\title{
THE “INTERNATIONALIST" ROSA LUXEMBURG AS A TRANSLATOR
}

\author{
Andreas F. Kelletat \\ Johannes Gutenberg University, Mainz, Germany
}

\begin{abstract}
This article is devoted to the description of the translation activity of Rosa Luxemburg, one of the most influential representatives of the German and European revolutionary left-wing Social Democracy. She was proficient in the Polish, Russian, German and French languages, and she conducted active propaganda, an important component of which consisted in the translation of works and articles by prominent Social Democrats into German and Polish. The author of the article carries out a diachronic analysis of the translation activity of Rosa Luxemburg. It is noted that translations from Russian and Polish played an important role in the preparation of the doctoral dissertation by Rosa Luxemburg. The dissertation was presented in Switzerland and dealt with the problems of Poland's industrial development. The translatological evaluation of Rosa Luxemburg's translations has not yet been undertaken, and the relevance of the given research consists in the new interpretation of the ways of forming the political discourse of the Social Democrats in Europe at the turn of the 19th and 20th centuries. Analysis of Rosa Luxemburg's main translations allows noting that the translation of Vladimir Korolenko's book The History of my Contemporary, which was published after her tragic death, is of the highest importance among all the translations. The translation of V. Korolenko's book was republished many times, and the prospect of the article consists both in evaluating the quality of R. Luxemburg's translation and in analyzing the perception of this book in Europe during the entire 20th century until 1985, when it was included in the Library of World Literature of the Zurich publishing house Manesse-Verlag.

Key words: Rosa Luxemburg, internationalist, social democrat, translator, translation, translation activity, propaganda, The History of my Contemporary.

Citation. Kelletat A.F. Die «Internationalistin» Rosa Luxemburg als Übersetzerin. Vestnik Volgogradskogo gosudarstvennogo universiteta. Seriya 2, Yazykoznanie [Science Journal of Volgograd State University. Linguistics], 2017, vol. 16, no. 3, pp. 41-47. DOI: https://doi.org/10.15688/jvolsu2.2017.3.4

УДК 81 '25:06.053.56

Дата поступления статьи: 10.07.2017

ББК 81.18

Дата принятия статьи: 25.08.2017
\end{abstract}

\section{«ИНТЕРНАЦИОНАЛИСТ» РОЗА ЛЮКСЕМБУРГ КАК ПЕРЕВОДЧИК}

\author{
Андреас Ф. Келлетат \\ Университет имени Йоханнеса Гутенберга, г. Майнц, Германия
}

\begin{abstract}
Аннотация. Статья посвящена описанию переводческой деятельности одной из наиболее влиятельных представительниц немецкой и европейской революционной левой социал-демократии - Розы Люксембург. Владевшая польским, русским, немецким и французским языками, она вела активную пропаганду, важной составляющей которой был перевод работ видных социал-демократов на немецкий и польский языки. В статье предпринят диахронический анализ переводческой деятельности Р. Люксембург. Отмечается, что переводы с русского и польского сыграли важную роль в подготовке защищенной в Швейцарии докторской диссертации ㄱ.Р. Люксембург, которая была посвящена промышленному развитию Польши. Транслатологическая оценка текстов переводов Р. Люксембург до сих пор не проводилась, и актуальность исследования, представленного в статье, заключается в новом осмыслении путей формирования политического дискурса социал-демократов в Европе рубежа XIX-XX веков. Анализ основных переводческих работ Р. Люксембург позволяет констатировать, что центральное место среди них принадлежит переводу работы Владимира Короленко «История моего современника», который был опубликован уже после трагической смерти переводчицы. В качестве перспективы исследования определяется необходимость оценки качества перевода, выполненного Р. Люксембург, и
\end{abstract}


анализ восприятия данного произведения в Европе в течение всего ХХ в., вплоть до 1985 г., когда он был включен в библиотеку мировой литературы цюрихского издательства «Manesse-Verlag».

Ключевые слова: Роза Люксембург, интернационалист, социал-демократ, переводчик, перевод, переводческая деятельность, пропаганда, «История моего современника».

Цитирование. Kelletat A. F. Die «Internationalistin» Rosa Luxemburg als Übersetzerin // Вестник Волгоградского государственного университета. Серия 2, Языкознание. - 2017. - Т. 16, № 3. - С. 41-47. - DOI: https:// doi.org/10.15688/jvolsu2.2017.3.4

UDK 81'25:06.053.56

Eingereicht: 10.07.2017

BBK 81.18

Angenommens: 25.08.2017

\title{
DIE «INTERNATIONALISTIN» ROSA LUXEMBURG ALS ÜBERSETZERIN
}

\author{
Andreas F. Kelletat \\ Johannes Gutenberg University, Mainz, Deutschland
}

\begin{abstract}
Dieser Artikel konzentriert sich auf der Beschreibung der Übersetzung von einer der einflussreichsten Vertreterinnen der deutschen und europäischen revolutionären linken Sozialdemokratie Rosa Luxemburg. Sie beherrschte einwandfrei Polnisch, Russisch, Deutsch und Französisch und betrieb ganz aktiv Propaganda, ein wichtiger Bestandteil davon war die Übersetzung von Werken und Artikeln der prominenten Sozialdemokraten in Deutsch und Polnisch.

In dem Artikel führte der Autor die diachronische Analyse der Übersetzungstätigkeit von Rosa Luxemburg durch. Es wird darauf hingewiesen, dass die Übersetzungen aus dem Russischen und Polnischen eine wichtige Rolle bei der Herstellung der Doktorarbeit von Rosa Luxemburg gespielt haben. Ihre wissenschaftliche Arbeit behandelt sich mit Fragen der industriellen Entwicklung Polens. Translatorische Auswertung von Übersetzungen von Rosa Luxemburg ist noch nicht unternommen worden, die Relevanz der Arbeit besteht in dem neuen Verständnis der Bildungsmöglichkeiten des politischen Diskurses der Sozialdemokraten in Europa an der Wende des 19./20. Jahrhunderts. Als Ergebnis der Analyse der wichtigsten Übersetzungsarbeiten von Rosa Luxemburg kam der Autor zum Schluss, dass die Übersetzung des Werkes von Vladimir Korolenko „Geschichte meines Zeitgenossen”, die nach dem tragischen Tod von Rosa Luxemburg veröffentlicht wurde, an der Spitze liegt. Übersetzung des Werkes von V. Korolenko wurde mehrfach nachgedruckt, die Forschungsperspektive des Artikels besteht in der Auswertung der Qualität der Übersetzung von Rosa Luxemburg, sowie in der Analyse der Wahrnehmung des literarischen Werkes in Europa im Laufe des 20. Jahrhunderts, bis zum Jahre 1985, als es in die globale Bibliothek der Weltliteratur des Züricher Verlags Manesse-Verlag aufgenommen wurde.
\end{abstract}

Schlüsselwörter: Rosa Luxemburg, Internationalistin, Sozialdemokratin, Übersetzerin, Übersetzung, Übersetzungstätigkeit, Propaganda, „Geschichte meines Zeitgenossen”.

Citation. Kelletat A.F. Die «Internationalistin» Rosa Luxemburg als Übersetzerin. Vestnik Volgogradskogo gosudarstvennogo universiteta. Seriya 2, Yazykoznanie [Science Journal of Volgograd State University. Linguistics], 2017, vol. 16, no. 3, pp. 41-47. DOI: https://doi.org/10.15688/jvolsu2.2017.3.4

Dass die Nationalökonomin und Politikerin Rosa Luxemburg auch als herausragende Schriftstellerin Beachtung finden sollte, hat als erster Karl Kraus gefordert. 1920 trug er in Berlin eine längere Passage aus einem Brief vor, den Luxemburg 1917 aus dem Breslauer „Weibergefängnis” an Sophie Liebknecht geschrieben hatte. Noch nie habe auf seinen Lesungen ein Text solch tiefen Eindruck gemacht wie dieses ,im deutschen Sprachgebrauch einzige Dokument von Menschlichkeit und Dichtung". Luxemburgs Brief gehöre in deutsche Schulbücher aufgenommen, „zwischen Goethe und Claudius“ [Kraus, 1920, S. 5].
Auch wenn sich manch anderer Leser ihrer Gefängnisbriefe von deren sprachlichem Reichtum und der in ihnen erkennbar werdenden „anderen” Rosa Luxemburg anrühren ließen (etwa Paul Celan oder Johannes Bobrowski), hat sich Kraus' Forderung, sie als bedeutende Schriftstellerin ernst zu nehmen, nicht durchgesetzt. Die Beschäftigung mit ihrem Leben und Werk blieb auf das Politische fixiert, auf ihre Rollen als marxistische Theoretikerin und Vertreterin des proletarischen Internationalismus, als Antimilitaristin, als Frauenrechtlerin, als Kritikerin und Bewunderin Lenins, als 
Spartakistin [Badia, 2002], als Gründungsmitglied der KPD bzw. als Märtyrerin der Novemberrevolution.

Dieser Revolution verdankte Rosa Luxemburg das Ende ihrer seit April 1915 andauernden Haftzeit bzw. „Sicherheitsverwahrung“. Am 8. November 1918 wurde sie aus dem Gefängnis entlassen, zwei Tage später war sie zurück in Berlin, wo sie, nach der Niederschlagung des Spartakusaufstands, am 15. Januar 1919 ermordet wurde.

Drei Jahre und vier Monate hat Luxemburg während des ersten Weltkriegs in Haftanstalten verbracht. Diese Zeit nutzte sie ,zum Lesen, Denken und Schreiben", wie es in einem ihrer ersten Gefängnisbriefe heißt [Luxemburg, 1984, Bd. V, S. 51]. Was sie in diesen Jahren gelesen, worüber sie nachgedacht und was sie geschrieben hat, wurde in zahlreichen Beiträgen anhand ihrer in den Werkausgaben veröffentlichten Texte untersucht. Unbeachtet blieb bisher jedoch ihr übersetzerisches Euvre, darunter herausragend jenes Buch, an dem sie vom Sommer 1916 bis zum Herbst 1918 gearbeitet hat: die 554 Druckseiten umfassende deutsche Version des zwischen 1906 und 1908 entstandenen ersten Bandes der Erinnerungen von Wladimir Korolenko [Korolenko, 1919; Pross-Weerth, 1970; Riggenbach, 1985]. Dass die im März 1919 bei Paul Cassirer in Berlin erschienene Geschichte meines Zeitgenossen ebenso wie ihre Prosa-Übersetzungen aus dem Französischen nicht in Luxemburgs Gesammelte Werke aufgenommen wurden, zeugt von einer bestimmten Werk-Vorstellung der Herausgeber, einer Vorstellung, wonach Übersetzungen nicht zum Euvre jener Personen gehören, die sie geschrieben haben.

Dass Luxemburg für übersetzerisches Handeln gut gerüstet war, ergibt sich aus der Sprach- und Topobiographie: In ihrer aus der jüdischen Gemeinschaft stammenden, stark assimilierten Familie wurde Polnisch gesprochen und eifrig deutsche Literatur gelesen, Schiller vor allem [Hexelschneider, 2004; Brandt, 1990]. Die Schule besuchte sie in Warschau, Unterrichtssprache dort war das Russische. Schon mit neun Jahren soll sie russische und deutsche Gedichte ins Polnische übertragen haben [Laschitza, 2000, S. 25; Nettl, 1968, S. 67]. Thr Abiturzeugnis bescheinigte der 17-jährigen für die Sprachen Polnisch, Russisch, Deutsch und Französisch sehr gute Kenntnisse. Von 1889 bis 1898 lebte sie in Zürich, studierte dort in der Fremdsprache Deutsch, ging 1894 für ein Gastsemester nach Paris, kam 1898 nach Berlin, war 1905/06 für ein Dreivierteljahr in Polen, Russland und Finnland. Reisen führten sie zwischen 1900 und 1914 immer wieder von Berlin ins Ausland, etwa zu den Sozialistenkongressen in Paris, London, Amsterdam, Kopenhagen und Brüssel. Auf dem Stuttgarter Kongress (1907) dolmetschte sie auf einer Massenveranstaltung die Ansprache des französischen Antimilitaristen und Antinationalisten Hervé. Als der den deutschen Genossen vorwarf, dass sie Angst vor dem Gefängnis hätten, unterbrach ihn zur allgemeinen Heiterkeit seine Dolmetscherin Luxemburg (die 1904 in Zwickau und 1906 in Warschau selbst im Gefängnis gesessen hatte) mit einem Non! [Hirsch, 1969, S. 81]

Neben der Muttersprache Polnisch dürfte das Deutsche mit der Zeit ihre stärkste Sprache geworden sein, beide Sprachen benutzte sie für ihre Publikationen, Briefe schrieb sie auch auf Russisch und Französisch. Kenntnisse im Englischen muss sie sich nebenher angeeignet haben, sie las viele englische Romane im Original.

In welchem Umfang ihre Arbeit als Redakteurin für die 1893 bis 1896 im Pariser Exil produzierte Monatsschrift Sprawa Robotnicza („Arbeitersache“) übersetzerische Aktivitäten umfasste, ist bisher nicht dokumentiert. In den (auf Polnisch geschriebenen, oft mit russischen Einsprengseln durchsetzten) Briefen an ihren Mentor, Mitstreiter und langjährigen Geliebten Leo Jogiches ist häufig von Übersetzungen aus dem Französischen, Russischen und Deutschen die Rede.

Mit einer regelrechten Internationalisierungskampagne beginnt sie 1898 in der Sächsischen Arbeiter-Zeitung. Sie berichtet über die Sozialdemokratische Bewegung in den litauischen Gouvernements Rußlands [Luxemburg, 2014, Bd. VI, S. 111-113], über das ,gehässige System der Germanisierung” im Posener Gebiet [Luxemburg, 2014, Bd. VI, S. 117f], mehrmals über die Dreyfus-Affäre in Frankreich oder über das Wiedererscheinen sozialistischer Zeitungen in Italien [Luxemburg, 2014, Bd. VI, S. 169f]. Manche dieser Auslandsberichte sind überwiegend wortwörtliche Übersetzungen, etwa ihr Text Der Sozialismus auf Guadeloupe über den „Vollblutneger” Jean Hégésippe Legitimus, durch dessen Wahl in die 
französische Nationalversammlung „der Sozialismus in der Kolonie <... zu einer Macht geworden ist" [Luxemburg, 2014, Bd. VI, S. 129]. Luxemburgs Beitrag besteht zu 3/5 aus einer (von ihr als direktes Zitat kenntlich gemachten) Übersetzung eines in der Reveil $d u$ Nord erschienenen Artikels. Genauso verfährt sie in dem Bericht Murawjew in Paris, nur dass es dieses Mal keine französischen, sondern russische Zeitungstexte sind, aus denen sie für die Sächsische Arbeiter-Zeitung übersetzt hat [Luxemburg, 2014, Bd. VI, S. 239f]. Gleich 9/10 des Beitrags Antonio Labiola über Bernstein wurden von ihr aus dem Französischen übernommen [Luxemburg, 2014, Bd. VI, S. 262-265].

Umfang und Intensität dieser translatorischen Aktivitäten sind bisher kaum wahrgenommen, geschweige analysiert worden, auch weil die entsprechenden Texte nicht gesammelt und ediert waren bzw. sind. Undeutlich bleibt daher bis heute, wie stark Luxemburg stets für mindestens zwei Adressatenkreise geschrieben hat, einen deutschsprachigen und einen polnischsprachigen. Für die in Krakau zwischen 1902 und 1910 erscheinende Monatsrevue Przeglad Socjaldemokratyczny hatte sie den Ehrgeiz, ,in jeder Nummer einen Artikel der namhaften Vertreter der Auslandsparteien" zu bringen [Luxemburg, 2014, Bd. VI, S. 161] und oft war sie es selbst, die diese Texte übersetzte. Durch die Auswahl der Beiträger, der Themen und deren Perspektivierung wollte sie auch ihre eigenen parteipolitischen Positionen stärken, etwa in der Frage des Massenstreiks, des Revisionismus, des Kolonialismus, des Nationalismus usw.

Dass sie durch Herkunft und Bildungsgrad (promovierte Frau in einem Land, in dem das Frauenstudium noch verboten war!), durch ihre Vielsprachigkeit und gelebte Interkulturalität im patriarchalischen Milieu der deutschen Sozialdemokratie seit ihrem ersten furiosen Auftritt auf dem Stuttgarter Parteitag im Oktober 1898 eine ebenso bewunderte wie kritisch beäugte Ausnahmeerscheinung blieb, bedarf kaum weiterer Erforschung. Jedoch könnte ihr Gesamtwerk aus translationshistorischer Perspektive neu gesichtet werden, also gefragt werden, was sie eigentlich wann warum und für wen übersetzt hat. Dass hierfür mit einem relativ weiten (wenn auch nicht kulturwissenschaftlich-metaphorischen) Übersetzungsbegriff gearbeitet werden muss, ergibt sich bereits aus der Tatsache, dass sich z. B. in ihren Auslandsberichten die Grenzen zwischen wörtlicher Wiedergabe, Paraphrase und kommentierender Zusammenfassung nicht immer klar erkennen lassen.

Analoges gilt für ihre 1898 in Leipzig veröffentlichte Abhandlung Die industrielle Entwicklung in Polen [Luxemburg, 1970, Bd. I, S. 112-216]. Denn auch diese Züricher Dissertation ist ein Resultat umfangreicher translatorischer Tätigkeit.

Ziel der Doktorarbeit war es, westeuropäische Wissenschaftler, aber auch Politiker, mit der industriellen Entwicklung im „russische(n) Hauptteil Polens“ vertraut zu machen - ausgehend von der Grundannahme, „daß die politische Physiognomie, die historischen Schicksale eines Landes für uns ein Buch mit sieben Siegeln (sind), wenn wir nicht sein wirtschaftliches Leben mit allen sich daraus ergebenden sozialen Folgen kennen" [Luxemburg, 1970, Bd. I, S. 115]. Und dieses ,wirtschaftliche Leben" wird von ihr für die Jahre 1850 bis 1896 aus einer Fülle polnischer und russischer Quellen dargestellt, aus ,,vielfach einander widersprechenden statistischen Werken, polemischen Broschüren, Zeitungsnotizen, offiziellen und nicht offiziellen Berichten" [Luxemburg, 1970, Bd. I, S. 116]. Durch die nationalökonomische (und wie man aus heutiger Sicht hinzufügen müsste: auch kulturwissenschaftlichdiskursorientierte) Analyse kann sie die immer enger werdende Verflechtung zwischen der polnischen und russischen Industrie aufzeigen. Das politische Ziel der polnischen „Bourgeoisie“ sei keineswegs die nationale Unabhängigkeit, sondern ,die völlige Verzichtleistung auf die nationale Freiheit für das Linsengericht der russischen Absatzmärkte" [Luxemburg, 1970, Bd. I, S. 194]. Die Interessengleichheit der polnischen und russischen Unternehmer werde dann allerdings auch - so heißt es, gut marxistisch prophezeit - ein „Endresul-tat" erzeugen, das

in gleichem Maße von der russischen Regierung, der polnischen Bourgeoisie und den polnischen Nationalisten außer acht gelassen wird: die Vereinigung des polnischen und des russischen Proletariats zum künftigen Syndikus bei dem Bankrott zuerst der russischen Zarenherrschaft und dann der polnisch-russischen Kapitalherrschaft [Luxemburg, 1970, Bd. I, S. 211]. 
Für das letzte Kapitel ihrer Dissertation befasste sich Luxemburg mit den ökonomischen Interessen Rußlands im Orient, wo man ,sich bereits auf den Weltmarkt hinauswagen und selbst den anderen kapitalistischen Nationen (England, Frankreich, Deutschland; AFK) auf fremdem Boden die Stirne bieten“" wolle [Luxemburg, 1970, Bd. I, S. 198]. Dass der globale Wettkampf der europäischen Mächte um Absatzmärkte schließlich in einen militärischen Konflikt, in einen Weltkrieg münden muss, sagt die Dissertation noch nicht. Aber dieses Thema wird Luxemburg in den kommenden Jahren intensiv beschäftigen, einschließlich der Frage, wie sich die europäischen Arbeiterparteien - mit der mächtigen SPD an ihrer Spitze - angesichts dieser Bedrohung verhalten müssten.

Als es zum Schwur kam, in der Berliner Reichstagssitzung am 4. August 1914, wurde die jahrzehntelang hochgehaltene antikapitalistische und antinationalistische Losung von den Proletariern aller Länder, die sich vereinigen sollten, beiseite geschoben. Damit war die „Kapitulation der internationalen Sozialdemokratie” besiegelt, „ein Unglück für die Menschheit" - so Luxemburg in ihrer 1916 veröffentlichten Junius-Broschüre, die die Mär von der „Verteidigung des Vaterlandes” zu widerlegen versucht und den „chauvinistischen Rausch“, das „Delirium des Nationalismus“ beschreibt sowie den Preis benennt: „Die Dividenden steigen, und die Proletarier fallen":

Wie Nationalismus und Chauvinismus in das Denken und Fühlen eines einzelnen Menschen, eines sehr jungen zumal, eindringen können - das ist zentrales Thema in Korolenkos Erinnerungen. Aus der Perspektive des Kindes bzw. Gymnasiasten schildert er, wie sich, ,in meiner Seele ein ganz elementarer Kampf zweier verschiedener Strömungen ab(spielte): der romantisch-nationalistischen und der realistischsozialen“ [Korolenko, 1919, S. 462]. Nicht zuletzt dieser Aspekt dürfte es gewesen sein, der die AntiNationalistin Luxemburg dazu gebracht hat, das Buch zu übersetzen. In ihrer Einleitung zur Geschichte meines Zeitgenossen schreibt sie:

Korolenko ist eben seiner Abstammung nach Pole, Ukrainer und Russe zugleich, und schon als Kind mußte er dem Ansturm der drei „Nationalismen” stand halten, von denen jeder ihm zumutete, ,irgend jemanden zu hassen und zu verfolgen“. An der gesunden Menschlichkeit des Knaben scheiterten frühzeitig alle derartigen Versuchungen. Die polnischen Traditionen wehten ihn nur als letzter ersterbender Hauch einer geschichtlich überwundenen Vergangenheit an. Von dem ukrainischen Nationalismus fühlte sein gerader Sinn sich durch das Gemisch von maskeradenhaftem Geckentum und reaktionärer Romantik abgestoßen. Und die brutalen Methoden der offiziellen Russifizierungspolitik gegenüber den unterdrückten Polen wie den Unierten in der Ukraine waren eine wirksame Warnung vor dem russischen Chauvinismus für ihn.

Die einzelnen Etappen der Übersetzungsarbeit, das Drängen des Verlags und immer neue Verzögerungen (auch durch die Postzensur) lassen sich aus Luxemburgs Briefen rekonstruieren. Dass es viel Hin und Her gab, hatte auch mit ihren Zweifeln zu tun, ob sie wirklich für das Übersetzen eines so anspruchsvollen literarischen Werks hinreichend gerüstet sei [Kelletat, 2016].

Urteile über die Qualität literarischer Übersetzungen finden sich in ihrer Privatkorrespondenz häufiger: Die Übersetzung „eines alten französischen Sonettbüchleins durch Rainer Maria Rilke" bewertete sie 1918 als „meisterhaft“" [Luxemburg, 1975, Bd. V, S. 389]. Eine Anna Karenina-Übersetzung hingegen fand sie „haarsträubend” und sie holte dann gleich zu einem heftigen Rundumschlag aus:

Was ich irgend an Übersetzungen aus der russischen Literatur gelesen habe, es war immer ein arger Schund, denn diese Übersetzungen werden meist von russischen Hungerleidern mosaischer Konfession ausgeführt, die sich als solche einbilden, die deutsche Sprache zu kennen, dabei aber literarisch völlig ungebildet sind.

Literarische Unbildung, Nicht-Vertrautsein mit dem in der deutschen Literatur entwickelten Ausdrucksrepertoire, konnte man Luxemburg nicht nachsagen. Trotzdem hatte sie den Wunsch, ihre Übersetzung von Muttersprachlern gegenlesen zu lassen. Genutzt wurde dazu ein Freundes-Netzwerk, für dessen Funktionieren ihre Sekretärin Mathilde Jacob zuständig war. Am Ende allerdings wurde ihr die redigierende Zuarbeit der Freunde zu viel, sie wollte die Alleinherrschaft über ihr Werk zurück haben. Ende Mai 1918 schrieb sie: 
Nun zu Korolenko $<\ldots>$ Mit ist plötzlich klargeworden, da $ß$ ich es nicht zugeben darf, da $ß$ noch jemand an dem Manuskript feilt! Der Gedanke ist mir unerträglich, daß ich unter meinem Namen eine Arbeit herausgeben soll, die nicht bis zum Tipfelchen über dem i mein ist $<\ldots$.. $>$ Ich will herauskommen telle quelle mit allen Slawismen und sonstigen Schönheitsfehlern. Also bitte $<\ldots>$ gib einfach den ganzen $\mathrm{Kram}<\ldots>$ zum Druck $<\ldots>$

Der Name Korolenko wird in Luxemburgs Briefen am 9. August 1918 zum letzten Mal erwähnt, als sie Mathilde Jacob die aus der Berliner Königlichen Bibliothek entliehenen Korolenko-Bücher zurücksandte. Diese Bücher hatte sie für den Einleitungs-Essay benötigt, in dem sie nicht nur Korolenkos Gesamtwerk und seinen Ort in der russischen Literatur zu beschreiben versuchte, sondern auch „das fein vibrierende soziale Gewissen“ [Korolenko, 1919] als Grundzug der russischen Literatur insgesamt charakterisierte. Wie stark dieser Essay durch den Blick auf aktuelle politische und soziale Entwicklungen geprägt ist, zeigt ihr Vergleich zwischen Korolenkos Kindheits- und Jugenderinnerungen und Gorkis Meine Kindheit:

$<\ldots>$ nur wer Gorkis Lebenserinnerungen gelesen (hat), kann seinen (des russischen Proletariats; AFK) wunderbaren Aufstieg aus dieser sozialen Tiefe zur vollen Sonnenhöhe moderner Bildung, genialer Kunst und einer wissenschaftlich fundierten Weltanschauung ermessen. Auch darin sind Gorkis persönliche Schicksale symbolisch für das russische Proletariat als Klasse, das sich mitten aus dem Rauhen und Krassen der äußeren Unkultur des Zarenreiches durch die harte Schule des Kampfes in erstaunlich kurzer Zeit von zwei Jahrzehnten zur geschichtlichen Aktionsfähigkeit emporgearbeitet hat. Sicher ein unbegreifliches Phänomen dies für alle Kulturphilister, die gute Straßenbeleuchtung, pünktlichen Eisenbahnverkehr und saubere Stehkragen für Kultur sowie fleißiges Klappern der parlamentarischen Mühlen für politische Freiheit halten.

Der Abschnitt enthält ex negativo alles, was Luxemburg an der Führung der deutschen Sozialdemokratie zu kritisieren hatte: deren NichtBereitschaft zum Kampf und zur Aktionsfähigkeit (Stichwort: Ablehnung des Massenstreiks, Bewilligung der Kriegskredite), deren Auffassung, auf parlamentarischem statt revolutionärem Weg die Interessen der Arbeiter wahren zu können (Stichwort: Revisionismus) und deren nur noch rhetorische aber nicht mehr das tägliche politische Handeln leitende Ausrichtung an einer "wissenschaftlich fundierten Weltanschauung" (Stichwort: faktische Leugnung des „Endziels” Sozialismus und Akzeptieren des kapitalistischen Systems). Spürbar wird auch, wie fremd sie bei aller Bewunderung für die deutsche Höhenkamm-Kultur - den Deutschen und ihren Marotten (Ordnung, Sauberkeit, Pünktlichkeit) geblieben war. Wie ein Echo klingt ihr Spott über die „Kulturphilister“ auf das, was sie genau 20 Jahre zuvor, in einem ihrer ersten Briefe aus Deutschland, für Leo Jogiches formuliert hatte:

Hab keine Angst, ich werde mich hier schon nicht germanisieren, ich hasse Berlin und die Schwaben (= Deutsche; AFK) aus ganzer Seele, der Schlag soll sie treffen. Aber deutsch quassele ich schon wie Bismarck selbst [Luxemburg, 1970, Bd. I, S. 131].

Die Veröffentlichung der Geschichte meines Zeitgenossen wurde am 11. Dezember 1918 im Börsenblatt für den Deutschen Buchhandel angekündigt, aber die Auslieferung des Buches verzögerte sich um mehrere Monate. „Die Übersetzung Die Geschichte meines Zeitgenossen ist jetzt im Buchhandel erschienen,“ heißt es zehn Wochen nach Luxemburgs Ermordung in einem Brief Mathilde Jacobs. „Ich bekam 5 Belegexemplare von Cassirer.“

Wie die Korolenko-Übersetzung in der Weimarer Republik rezipiert und unter welchen Bedingungen bzw. in welchen Kontexten die Geschichte meines Zeitgenossen nach 1945 mehrfach neu herausgegeben wurde - bis zu ihrem Ritterschlag durch die Aufnahme in die Weltliteratur-Bibliothek des Züricher ManesseVerlags 1985, soll an anderer Stelle dargelegt werden. Dort wird es auch um die Frage gehen, wie genau Rosa Luxemburg mit der russischen Vorlage verfahren ist.

\section{LITERATURLISTE}

Badia G. Rosa Luxemburg. Aus dem Französischen von Klaus Fritz [Rosa Luxemburg. From the French by Klaus Fritz]. Etienne François, Hagen Schulze (Hg.) Deutsche Erinnerungsorte II [German Remembrance Places II]. Munich, C. H. Beck Verlag, 2002,pp. 105-121. 
Brandt W. Rosa Luxemburg [Rosa Luxemburg]. Hans Jürgen Schultz (Hg.) Es ist ein Weinen in der Welt. Hommage für deutsche Juden unseres Jahrhunderts [There is a Cry in the World. Tribute to German Jews of our Century]. Stuttgart, Quell Verlag, 1990, pp. 100-119.

Hexelschneider E. Rosa Luxemburg und die Künste [Rosa Luxemburg and the Arts]. Leipzig, RosaLuxemburg-Stiftung Sachsen, 2004. 230 p.

Hirsch H. Rosa Luxemburg in Selbstzeugnissen und Bildern [Rosa Luxemburg in Self-Testimony and Pictures]. Reinbek at Hamburg, Rowohlt Verlag, 1969. 160p.

Kelletat A.F. Lesen, Denken und Schreiben Rosa Luxemburg als Übersetzerin [Reading, Thinking and Writing - Rosa Luxemburg as a Translator]. Julia Richter, Larisa Schippel (Hg.) (Neu-)Kompositionen. Aspekte transkultureller Translationswissenschaft [(Re) compositions. Aspects of transcultural translation science]. Berlin, Frank\&Timme Verlag, 2016, pp. 151-178.

Korolenko W. Die Geschichte meines Zeitgenossen [The Story of my Contemporary]. Aus dem Russischen übersetzt und mit einer Einleitung versehen von Rosa Luxemburg. 2 Bänder. Berlin, Paul Cassirer Verlag, 1919.

Kraus K. Vorlesungen (mit dem Brief Rosa Luxemburgs) [Lectures (with the Letter of Rosa Luxemburg)]. Die Fackel, 1920, no. 546-550, pp. 5-9.
Laschitza A. Im Lebensrausch trotz alledem. Rosa Luxemburg. Eine Bibliographie [In life, in spite of Everything. Rosa Luxemburg. A Bibliography]. Berlin, Aufbau Verlag, 2000. 688 p.

Luxemburg R. Gesammelte Briefe [Collected Letters]. Von Annelies Laschitza (Hg.) Leitung der Redaktion: Annelies Laschitza und Günter Radzun. Bd. V. Berlin, Dietz Verlag, 1984.

Luxemburg R. Gesammelte Werke [Collected Works]. Bd. I-V. Leitung der Redaktion: Dr. Günter Radzun. Berlin, Dietz Verlag, 1970-1975; Bd. VI: 1893 bis 1906. Hg. u. bearb. von Annelies Laschitza und Eckhard Müller. Berlin, Aufbau Verlag, 2014.

Nettl P. Rosa Luxemburg [Rosa Luxemburg]. Aus dem Englischen (1965) von Karl Römer. 2. Aufl. Cologne, Berlin, Kiepenheuer und Witsch Verlag, 1968. 576 p.

Pross-Weerth H. Korolenko, Wladimir: Die Geschichte meines Zeitgenossen [Rezension] [Korolenko, Wladimir: The History of my Contemporary (Review). Frankfurter Allgemeine Zeitung, 1970, July 18, p. 5.

Riggenbach H. Nachwort zu unserer Ausgabe [Epilogue to our Edition]. Wladimir Korolenko: Die Geschichte meines Zeitgenossen [Vladimir Korolenko: The History of my Contemporary]. Aus dem Russischen übersetzt von Rosa Luxemburg. Zurich, Manesse Verlag, 1985, pp. 631-647.

\section{Information about the Author}

Andreas F. Kelletat, Doctor, Professor, Head of the Department of German Language and Intercultural German Studies, Institute of Translation Studies, Linguistics and Cultural Studies, The Johannes Gutenberg University of Mainz, An der Hochschule, 2, D-76726 Germersheim, Mainz, Germany, kelletat@uni-mainz.de, idn=122945212 (Deutsche National Bibliothek)

\section{Информация об авторе}

Андреас Ф. Келлетат, доктор, профессор, руководитель отделения немецкого языка и межкультурной германистики, Институт переводоведения, языкознания и культурологи, Университет имени Йоханнеса Гутенберга, Ан дер Хохшуле, 2, D-76726 Гермерсхайм, г. Майнц, Германия, kelletat@uni-mainz.de, idn=122945212 (Немецкая национальная библиотека)

\section{Information über den Autor}

Andreas F. Kelletat, Univ.-Prof. Dr. Dr. h. c. mult., Arbeitsbereich Deutsch / Interkulturelle Germanistik, Arbeitsbereichsleitung, Universitätsprofessor Fachbereich 06: Translations-, Sprach- und Kulturwissenschaft (FTSK), Johannes Gutenberg-Universität Mainz, An der Hochschule, 2, 76726 Germersheim, Mainz, Deutschland, kelletat@uni-mainz.de, idn=122945212 (Deutsche National Bibliothek) 